\title{
Dexmedetomidine in prevention and treatment of postoperative and intensive care unit delirium: a systematic review and meta-analysis
}

Julian Flükiger ${ }^{1 \dagger}$, Alexa Hollinger ${ }^{1,2,3^{*}+}$, Benjamin Speich ${ }^{4,5}$, Vera Meier ${ }^{1}$, Janna Tontsch ${ }^{1}$, Tatjana Zehnder ${ }^{1}$ and Martin Siegemund ${ }^{1}$

\begin{abstract}
Background: To determine the preventive and therapeutic effect of dexmedetomidine on intensive care unit (ICU) delirium.

Methods: The literature search using PubMed and the Cochrane Central Register of Controlled Trials was performed (August 1,2018 ) to detect all randomized controlled trials (RCTs) of adult ICU patients receiving dexmedetomidine. Articles were included if they assessed the influence of dexmedetomidine compared to a sedative agent on incidence of ICU delirium or treatment of this syndrome. Accordingly, relevant articles were allocated to the following two groups: (1) articles that assessed the delirium incidence (incidence comparison) or articles that assessed the treatment of delirium (treatment comparison). Incidence of delirium and delirium resolution were the primary outcomes. We combined treatment effects comparing dexmedetomidine versus (1) placebo, (2) standard sedatives, and (3) opioids in random-effects meta-analyses. Risk of bias for each included RCT was assessed following Cochrane standards.

Results: The literature search resulted in 28 articles ( 25 articles/4975 patients for the incidence comparison and three articles/166 patients for the treatment comparison). In the incidence comparison, heterogeneity was present in different subgroups. Administration of dexmedetomidine was associated with significantly lower overall incidence of delirium when compared to placebo (RR 0.52; 95\% Cl 0.39-0.70; $P^{2}=37 \%$ ), standard sedatives (RR 0.63; 95\% Cl 0.46-0.86; $P^{2}=69 \%$ ), as well as to opioids (RR $0.61 ; 95 \% \mathrm{Cl} 0.44-0.83 ; P^{2}=0 \%$ ). Use of dexmedetomidine significantly increased the risks of bradycardia and hypotension. Limited data were available on circulatory insufficiency and mortality. In the treatment comparison, the comparison drugs in the three RCTs were placebo, midazolam, and haloperidol. The resolution of delirium was measured differently in each study. Two out of the three studies indicated clear favorable effects for dexmedetomidine (i.e., compared to placebo and midazolam). The study comparing dexmedetomidine with haloperidol was a pilot study $(n=20)$ with high variability in the results.
\end{abstract}

Conclusion: Findings suggest that dexmedetomidine reduces incidence and duration of ICU delirium. Furthermore, our systematic searches show that there is limited evidence if a delirium shall be treated with dexmedetomidine.

Keywords: Delirium, Dexmedetomidine, Meta-analysis

\footnotetext{
*Correspondence: alexa.hollinger@usb.ch

${ }^{\dagger} J$ ulian Flükiger and Alexa Hollinger have contributed equally to study design and writing of the manuscript

${ }^{1}$ Department for Anesthesia, Surgical Intensive Care, Prehospital Emergency Medicine and Pain Therapy, University Hospital Basel, Spitalstrasse 21, 4031 Basel, Switzerland

Full list of author information is available at the end of the article
}

\section{Springer Open}

(c) The Author(s) 2018. This article is distributed under the terms of the Creative Commons Attribution 4.0 International License (http://creativecommons.org/licenses/by/4.0/), which permits unrestricted use, distribution, and reproduction in any medium, provided you give appropriate credit to the original author(s) and the source, provide a link to the Creative Commons license, and indicate if changes were made. 


\section{Background}

Delirium is experienced in $20 \%$ to $40 \%$ of the critically ill and up to $80 \%$ of mechanically ventilated (MV) medical or surgical patients [1-4]. It is a confusional state that has been described as a transient global disorder of cognition, awareness, and attention and as such is not only challenging for the treating medical team, but also has a considerable impact on affected patients. It is associated with prolonged hospital length of stay and time on MV, deterioration in cognition, and increased morbidity and mortality causing additional health-care expenses [4-6]. Its pathophysiological mechanisms are highly heterogeneous-as perceived by the high number of risk factors [7] - and have yet to be fully understood. Imbalanced neurotransmitter systems such as the reduction of acetylcholine activity, excess of serotonin and dopamine, or the release of gamma-amino-butyric acid (GABA) seem essential. Certain hospital care characteristics (patient immobilization, bladder catheter, or sleep deprivation) further contribute to the development of delirium $[8,9]$.

Treatment approach with sedation offers several benefits: First, it reduces discomfort, anxiety, and stress. Second, it facilitates the daily intensive care unit (ICU) procedures for the treating nurses and physicians, providing a calm, cooperative patient who is easy to rouse and capable of communicating pain and other needs [10-12]. So far, there is no ideal sedative agent that fulfills the criteria of being cheap, rapid in onset and offset, and without local or systemic adverse effects [10].

Although the 2013 PAD guidelines suggested against the use of antipsychotics such as haloperidol for the treatment of delirium due to their side effects (e.g., extrapyramidal symptoms, neuroleptic malignant syndrome, and QTc interval prolongation), they are still commonly applied today $[1,13,14]$. Moreover, the most recent 2013 American College of Critical Care Medicine Guidelines for the management of pain, agitation, and delirium (PAD) in adult ICU patients recommend the use of a non-benzodiazepine-based sedation approach [15].

Dexmedetomidine with its broad range of effects including easily controllable sedation, analgesia, and anxiolysis still enables the caring medical team to interact with the patient. It reduces the activity while still maintaining the reactivity of neurons in the locus coeruleus. Therefore, it is an appealing alternative to traditional sedatives such as propofol and benzodiazepines [16]. As a highly selective $\propto_{2}$-receptor agonist with no effect on the GABA receptor, it interacts with transmembrane G-protein-binding adrenoreceptors in the periphery $(\alpha$ $\left.{ }_{2 \mathrm{~A}}\right)$, as well as in the brain $\left(\alpha_{2 \mathrm{~B}}\right)$ and the spinal cord $\left(\propto_{2 \mathrm{C}}\right)$. Inducing a sleep-like state without respiratory depression may explain the beneficial effects of dexmedetomidine $[17,18]$, since disturbed circadian rhythm is a known contributing factor of delirium [19]. These characteristics lead to an easily arousable, communicative, and cooperative patient and render dexmedetomidine a potential therapeutic option for the ICU delirium, in addition to its suggested use for delirium prevention [16, 20]. However, inhibition of sympathetic activity in the periphery leads to sequential decreases in blood pressure and heart rate [19], the most commonly reported adverse events associated with dexmedetomidine [21].

A review from 2013 suggested dexmedetomidine could be suitable for both prevention and treatment of ICUassociated delirium [14]. In 2014, a systematic review from Pasin et al. [20] including 14 RCTs with a total of 3029 patients demonstrated that use of dexmedetomidine for anesthesia and sedation was associated with a significant reduction of the incidence of delirium. Similar results were demonstrated in a previous review from Xia et al. where only propofol was used as a comparator [22]. Very recently, Duan et al. [23] performed a meta-analysis, showing that dexmedetomidine can reduce postoperative delirium incidence in adult cardiac and non-cardiac surgery patient. Based on fact that in the meantime a number of additional randomized controlled trials (RCTs) were published, we wanted to provide an up-to-date meta-analysis on dexmedetomidine for both treatment and prevention of ICU delirium to define areas of future research concerning ICU delirium and dexmedetomidine treatment. Randomized controlled trials (RCTs) were included, assessing the effect of dexmedetomidine on delirium in adult ICU patients. The goal was to summarize current evidence on the potential of dexmedetomidine to lower the incidence and duration of ICU delirium.

\section{Methods}

For this systematic review and meta-analysis, we adhered to the PRISMA statement for reporting systematic reviews and meta-analyses [24].

\section{The literature search}

Authors performed an electronic database search of PubMed and the Cochrane Central Register of Controlled Trials (CENTRAL). The detailed search strategy is available in appendix (Additional file 1: Appendix). We used standard filters to search for RCTs. The last date of search was August 1, 2018.

\section{Inclusion criteria}

Two independent investigators (JF and AH) identified all potentially relevant studies in PubMed and CENTRAL based on a screening of titles and abstracts. Of these, full texts were obtained and reviewed for eligibility by the same two investigators. Any conflict of opinion was resolved by consensus with a third reviewer (MS). 
All studies that met the following inclusion criteria were selected: (1) RCT; (2) adult ( $\geq 18$ years) medical or surgical ICU patients; (3) sedation with dexmedetomidine versus any comparator either for prevention or treatment of ICU delirium, regardless of dose, duration, or time of administration; (4) incidence of delirium as a mandatory outcome measurement regarding incidence comparison; and (5) full text available in English. The exclusion criteria were duplicate publications, missing indication of delirium incidence, study design other than $\mathrm{RCT}$, focus on withdrawal delirium, oral administration of dexmedetomidine, or dexmedetomidine administered in both intervention and control groups.

\section{Data extraction}

Study-relevant information was extracted by two independent investigators (JF and $\mathrm{AH}$ ) for each included RCT. Any conflict of opinion was resolved by consensus with a third reviewer (MS).

Interventional (dexmedetomidine) and control drugs, publication date, study location and date of study conduct, as well as patient characteristics, total number of patients, and conducted procedure were considered relevant for data extraction. Control drugs were divided into three groups: placebo, standard sedatives (including propofol, midazolam, and lorazepam), and opioids (including morphine and remifentanil). Delirium assessment tools (e.g., confusion assessment method for the $\mathrm{ICU}=\mathrm{CAM}-\mathrm{ICU}$ or the intensive care delirium screening checklist $=$ ICDSC) were assessed. Furthermore, we recorded if delirium was assessed as the primary, a secondary, or no specific endpoint.

Although our inclusion criteria focused only on the incidence of delirium, we also conducted further analyses of other patient-relevant factors, such as adverse events (i.e., mortality, bradycardia, tachycardia, hypotension, hypertension, and circulatory insufficiency) and clinical outcome data (i.e., ICU length of stay, and time to extubation or duration of MV). These analyses have only an explorative character and raise no claim to completeness.

According to whether dexmedetomidine and control drugs were applied for treatment or prevention of ICU delirium, included trials were assigned to incidence comparison or delirium treatment (treatment comparison) and analyzed according to their affiliation.

For assessment of adverse events, we also considered studies reporting adverse events during anesthesia and subsequent intensive care sedation to increase the reliability of the study since only a few studies reported adverse events during intensive care sedation with dexmedetomidine.

The primary outcome measure in incidence comparison was incidence of delirium. The primary outcome measure in treatment comparison was delirium resolution.

\section{Risk of bias assessment}

Risk of bias for each included RCT was assessed using the Cochrane Collaboration's tool [25]. Two investigators (JF and BS) reviewed each RCT for risk of bias considering the following five key domains: (1) random sequence generation (selection bias); (2) allocation concealment (selection bias); (3) blinding of participants and personnel (performance bias); (4) blinding of outcome assessment (detection bias); and (5) incomplete outcome data (attrition bias). Each domain was judged as "low risk," "high risk," or "unclear risk" of bias. Any disagreement in opinion was resolved by consensus. We estimated the overall risk of bias for a RCT as low if the risk of bias was low in all key domains, as unclear if the risk of bias was unclear in at least one key domain, and as high if the risk of bias was high in at least one key domain.

\section{Statistical analysis}

Meta-analyses were conducted using the Cochrane Review Manager 5 (RevMan5). Differences in binary outcomes were presented as risk ratios (RR) including 95\% confidence intervals (CI) based on random and fixed effect models. RRs below 1 indicate that the event occurred less often in patients who received dexmedetomidine compared to patients who received a control drug. In incidence comparison, meta-analyses were performed to compare the incidence of delirium, adverse events, and clinical outcomes for each control drug separately. For the incidence of delirium, the following subgroup and sensitivity analyses were conducted: analyzing only studies with low risk of bias according to risk of bias assessment, population (i.e., cardiac surgery, MV, and non-cardiac surgery), and using a trim-and-fill method $[26,27]$ if there was an indication of publication bias.

Higgin's $I^{2}$ was conducted to assess the heterogeneity of the studies [28]. If a subgroup consisted of sufficient RCTs, Egger's test was performed and funnel plot was visually assessed to determine the risk of publication bias. Symmetric statistics and trim-and-fill methods were conducted using $\mathrm{R}$ version 3.4.1 (meta package) following the Cochrane recommendations [29]. Furthermore, metaanalyses were conducted for the following adverse events and clinical outcomes: mortality, bradycardia, tachycardia, hypotension, hypertension, circulatory insufficiency, ICU length of stay, and time to extubation or duration of MV. If both time to extubation and duration of MV were presented, we used time to extubation data for the metaanalyses. In case no means and standard deviations (SDs) were reported (for ICU length of stay, time to extubation or duration of $\mathrm{MV}$ ), we used medians, interquartile 
ranges (IQRs), or ranges to estimate these attributes, forcing us to make several assumptions (Additional file 1: Tables S1-S3) [30, 31].

Mortality rates assessed up to 45 days and the remaining adverse events were pooled regardless of the time they occurred (intraoperatively, postoperatively, during sedation or MV).

For treatment comparison, all articles reported different outcomes. Therefore, results are summarized descriptively and no meta-analysis was performed.

\section{Results}

\section{Trial identification}

After excluding duplicate studies, our literature search on PubMed and CENTRAL retrieved 232 articles. By screening titles and abstracts, 171 publications were excluded at the title abstract level and an additional 33 were excluded after consulting the full text (Fig. 1). The remaining 28 articles were included and divided into incidence comparison consisting of 25 RCTs [32-56] and treatment comparison consisting of three articles [5759] (Fig. 1).

\section{Study characteristics}

In incidence comparison, all RCTs were published between 2005 and 2018 (being conducted between 2002 and 2017; Table 1). Two RCTs did not state study dates $[39,40]$. Sixteen RCTs dealt with surgical patients, nine of which were cardiac surgery RCTs [36, 39, 41, 42, 45$47,51,53]$ and seven non-cardiac surgery RCTs, meaning microvascular free flap surgery [34], joint replacement [49] or total hip arthroplasty [54], major abdominal surgery [52], major laparoscopic surgery [55], and elective

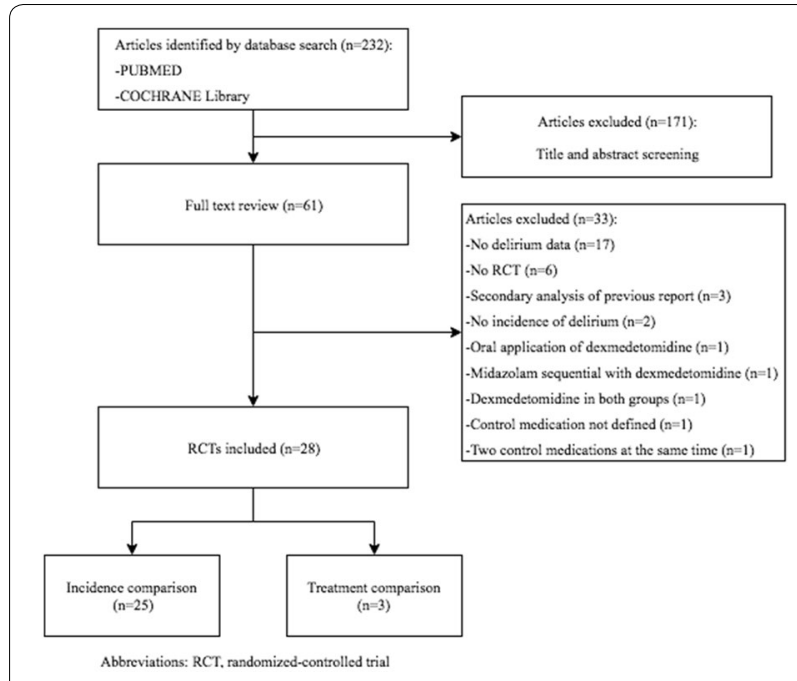

Fig. 1 Flow diagram of the systematic search for RCTs non-cardiac surgery under general anesthesia [33, 48]. The remaining nine RCTs consisted of six studies examining MV patients [32, 38, 40, 43, 44, 50] two examining noninvasively ventilated patients $[35,37]$ and one where dexmedetomidine was used for nightly sedation [56]. Applied procedures for administering intervention and control drugs were highly heterogeneous (Table 1). Comparators were placebo in eight RCTs [33-35, 45, 48, 51, $55,56]$, propofol in six RCTs [42, 46, 47, 52-54], midazolam in two RCTs [37, 50], and lorazepam [32], morphine [41], and remifentanil [36] in one RCT each. All of these RCTs used a two-arm design. Two RCTs compared dexmedetomidine sedation to a sedation regimen of either propofol or midazolam [43, 44]. Two RCTs used a three-arm design comparing dexmedetomidine to midazolam and propofol $[39,40]$. One RCT used a twoarm design comparing dexmedetomidine to placebo, but did not report overall results. Instead, only results for the two subgroups "normal-aged" patients and patients with amnestic mild cognitive impairment (aMCI) after joint replacement were reported [49]. One article consisted of two separate RCTs comparing dexmedetomidine to midazolam and propofol, respectively [38]. The total number of patients in incidence comparison was 4975. The used tools for delirium assessment are listed in Table 1. In treatment comparison, all RCTs were published between 2009 and 2016 (study dates ranged from 2005 to 2013; Table 1). Study populations of all RCTs consisted of patients with agitated delirium. Two RCTs dealt with MV patients in whom extubation was considered unsafe because of agitated delirium $[57,59]$. One RCT examined patients presenting with delirium following extubation failure after cardiac surgery [58]. Interventional and control drugs were administered according to the desired RASS score $[57,59]$, except for one RCT, where they were administered according to blood pressure and heart rate [58]. Comparators were placebo [57], midazolam [58], or haloperidol [59]. The total number of patients in treatment comparison was 166. Delirium assessment was performed using validated tools (Table 1).

Further information on the drug doses used throughout the studies is provided in Additional file 2: Table S4.

\section{Quality of evidence}

The Cochrane Collaboration's risk of bias assessment is provided in Table 2. Six [56] articles consisting of seven RCTs in the incidence comparison had low risk of bias [33-35, 38, 45]. Nine RCTs had an unclear risk of bias in at least one domain due to poor reporting (1808 patients) [32, 36, 37, 40, 41, 48, 50, 51, 53], and ten RCTs had a high risk of bias in at least one of the five assessed domains [39, 42-44, 46, 47, 49, 52, 54, 55]. In the treatment 


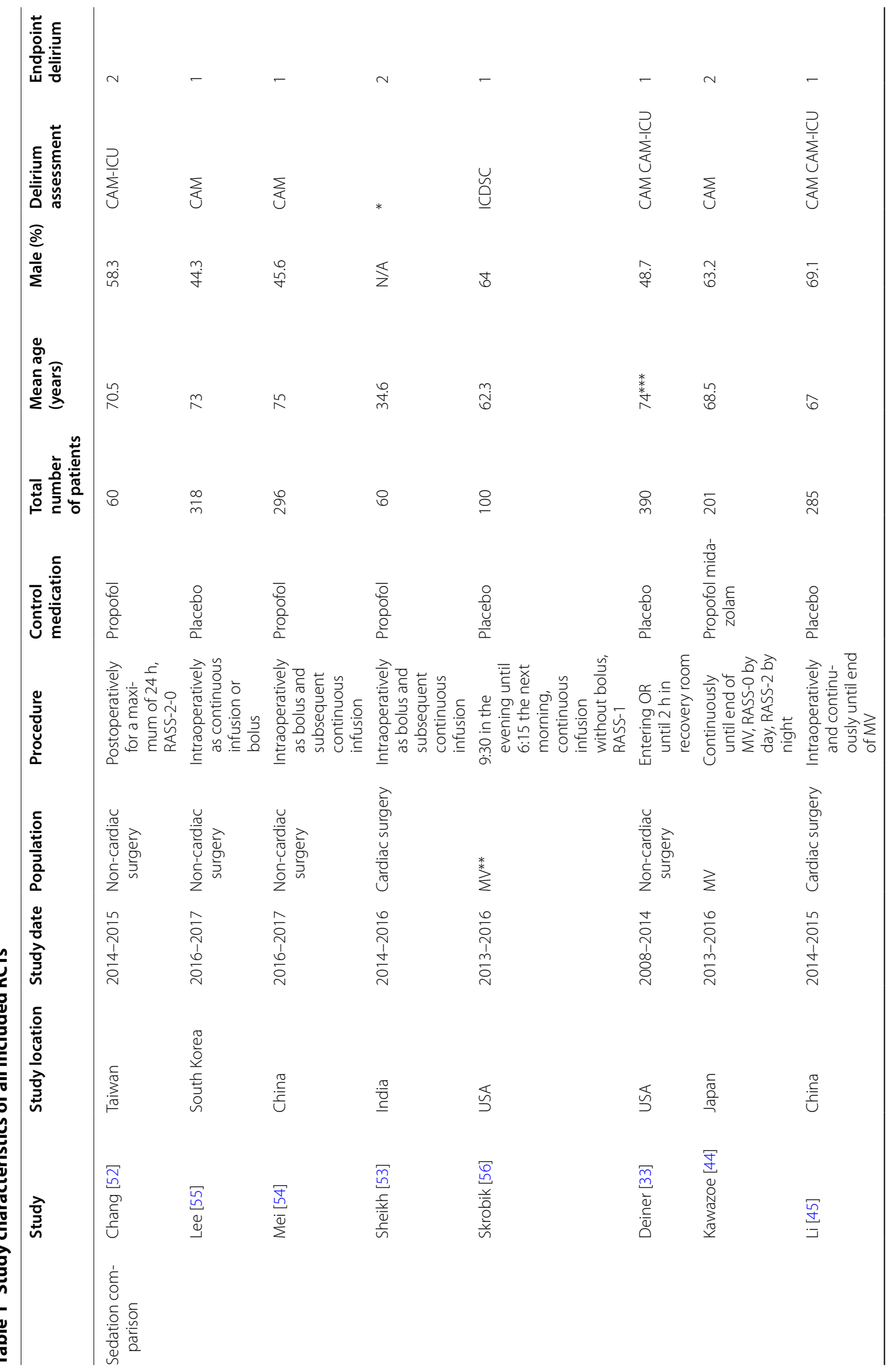




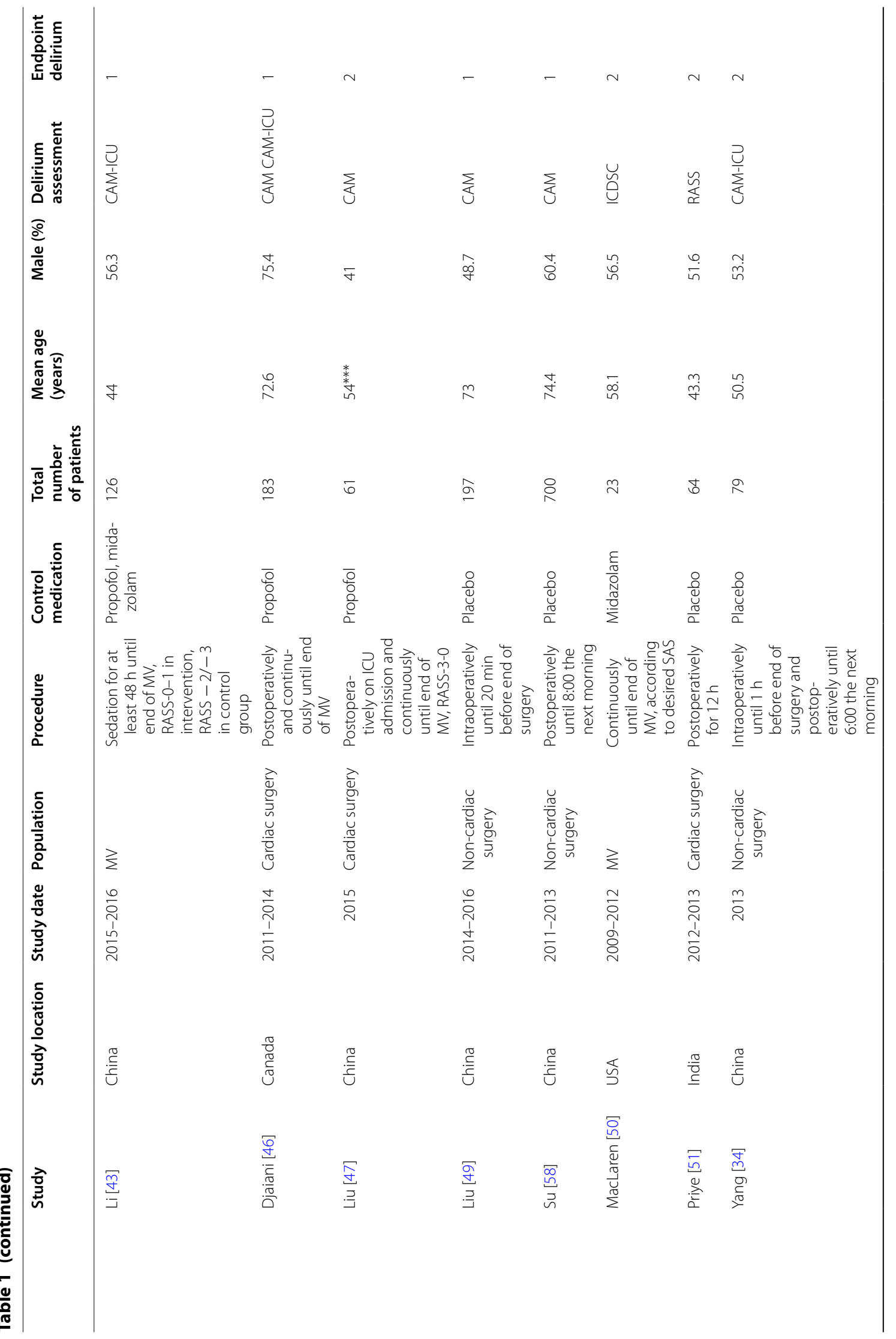


Flükiger et al. Ann. Intensive Care (2018) 8:92

Page 7 of 15

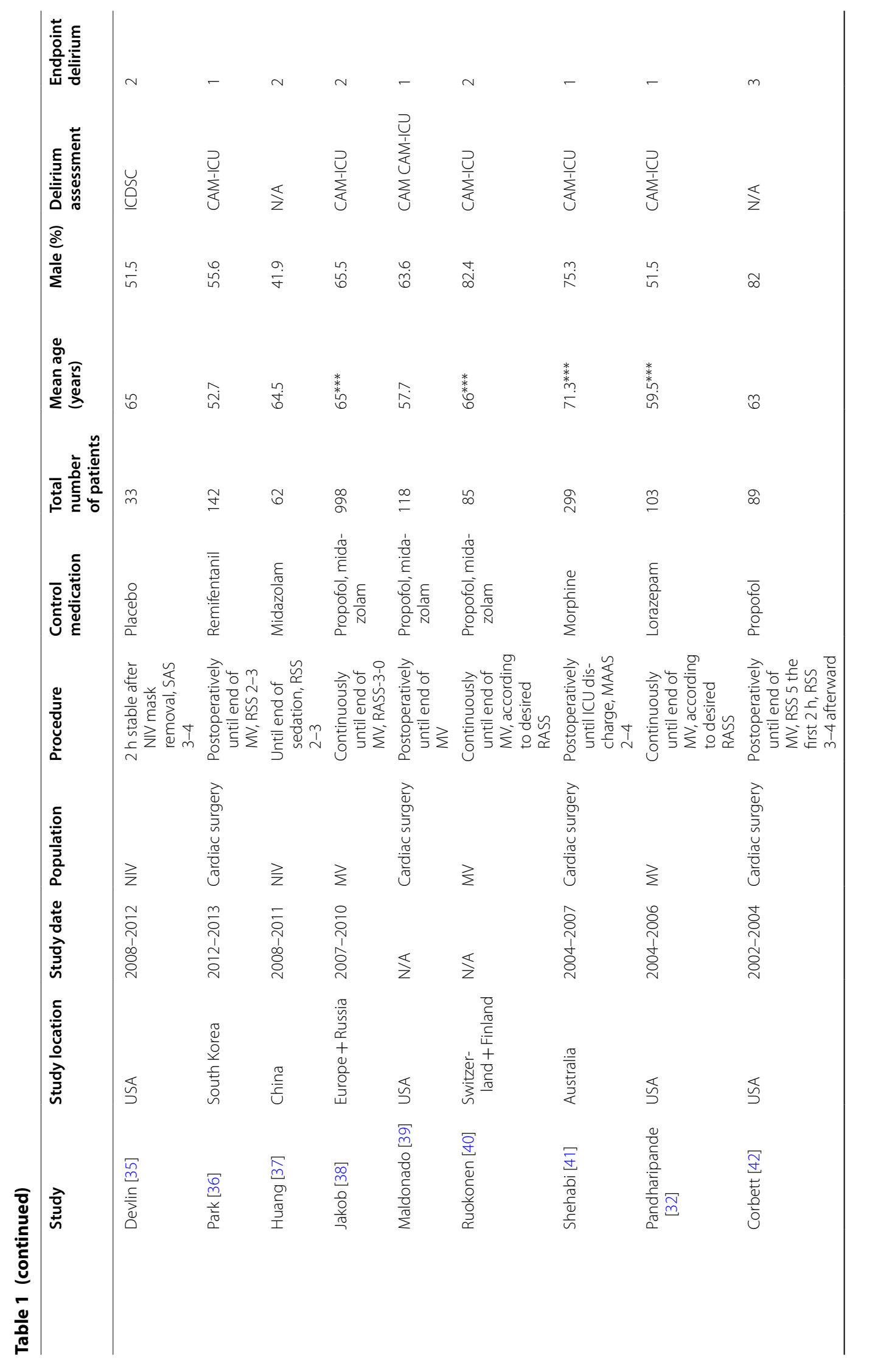




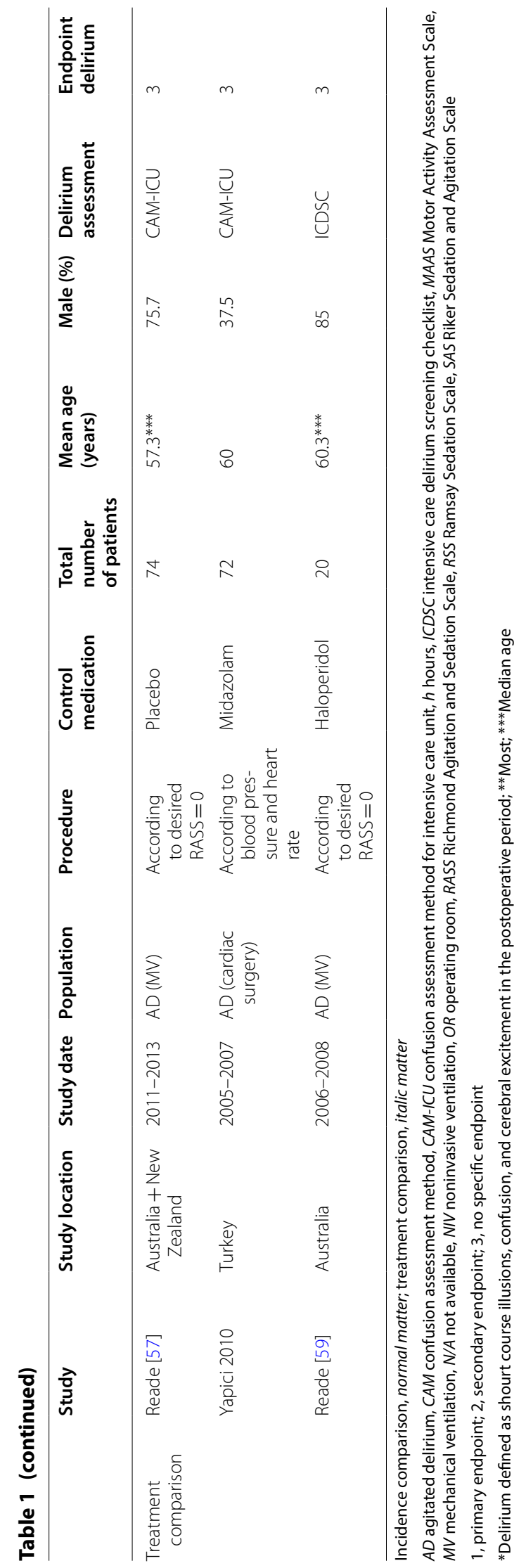


Table 2 Risk of bias assessment of included RCTs

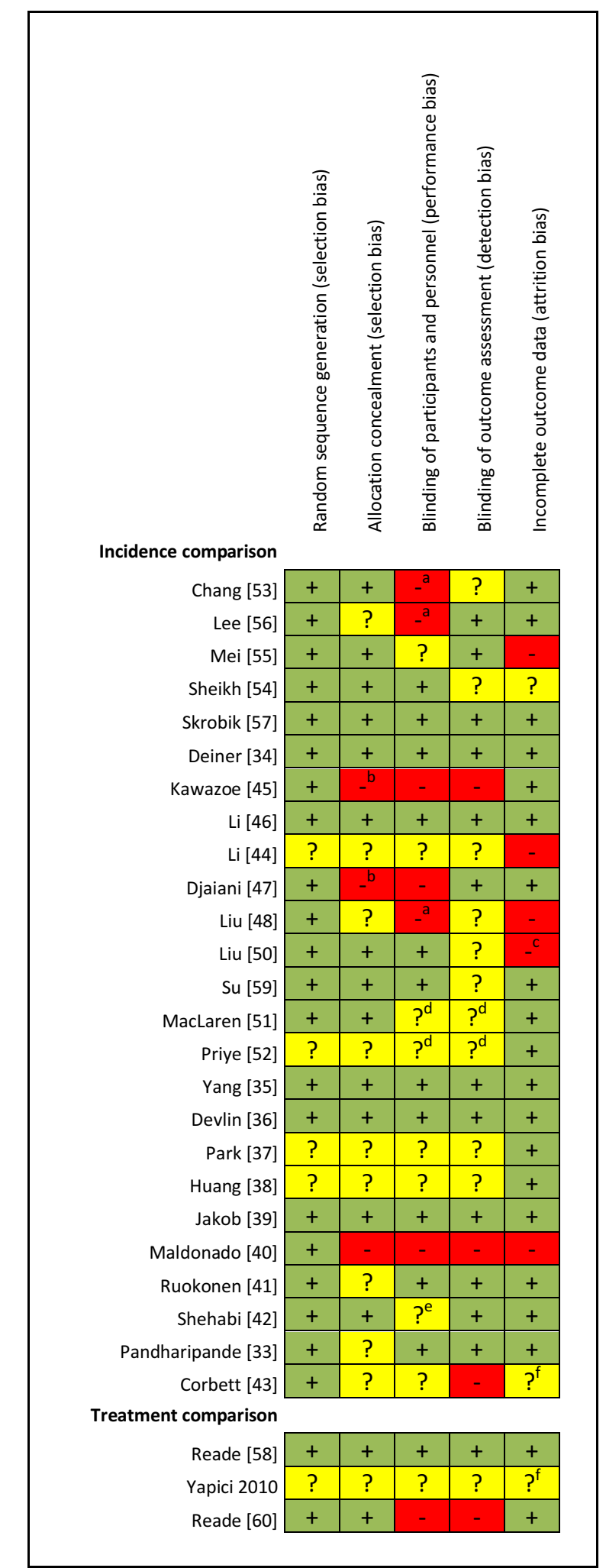

Table 2 (continued)

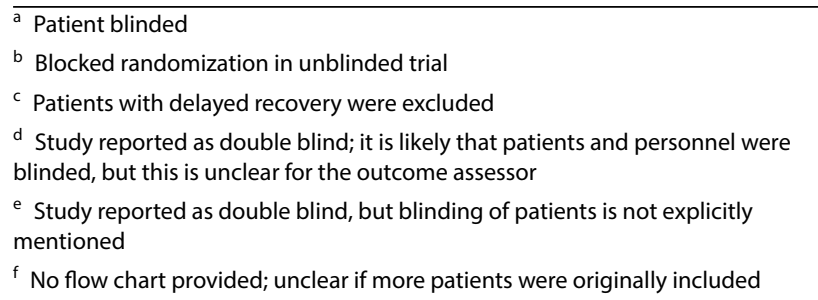

comparison, there was one RCT with low risk of bias [57], one with unclear risk of bias in all five domains [58], and one with high risk of bias in two of the five domains [59].

\section{Incidence comparison}

Nine articles consisting of ten data sets compared dexmedetomidine to placebo; 14 articles consisting of 15 data sets compared dexmedetomidine to standard sedatives; and two RCTs (two data sets) compared dexmedetomidine to opioids (Table 3).

\section{Incidence of delirium}

Overall incidence of delirium in the dexmedetomidine group was significantly lower compared to placebo (RR 0.52 ; $95 \%$ CI $0.39-0.70 ; I^{2}=37 \%$; Fig. 2), compared to standard sedatives (RR 0.63; 95\% CI 0.46-0.86; $I^{2}=69 \%$; Fig. 3), as well as compared to opioids (RR 0.61; 95\% CI $0.44-0.83 ; I^{2}=0 \%$; Fig. 4$)$. When only RCTs with a low risk of bias were included, we retrieved a RR of 0.66 (95\% CI 0.43-1.03; five data sets included) for the placebo comparison and a RR of 0.64 (0.41-0.98; two data sets included) for the standard sedative comparison (Table 3). It is important to mention that the two data sets from the standard sedatives comparison were presented in the same article [38]. In the opioids group, there was no RCT without risk of bias.

Visual inspection of the funnel plot from the analysis of standard sedatives reveals some deviation from the funnel shape (Additional file 1: Figure S1), indicating the presence of publication bias (Egger's test: $p=0.015$ ). This was, however, not the case for the placebo comparison (Egger's test: $p=0.62$; Additional file 1: Figure S2).

Results from the trim-and-fill method (dexmedetomidine vs. standard sedatives) were less clear about the favorable effect compared to the overall result (RR 0.77; 95\% CI 0.57-1.05; Table 3). Further subgroup and sensitivity results are presented in Table 3 . Considerable heterogeneity was found in the subgroup analysis. 
Table 3 Subgroup and sensitivity analyses for incidence of delirium

\begin{tabular}{|c|c|c|c|c|}
\hline Subgroup and sensitivity analyses & Number of data sets & Number of patients & $\begin{array}{l}\text { Risk ratio }(95 \% \mathrm{Cl}) \text { random effect } \\
\text { model }\end{array}$ & $I^{2}$ \\
\hline \multicolumn{5}{|l|}{ Placebo } \\
\hline Overall & 10 & 2071 & $0.52(0.42-0.63)$ & $37 \%$ \\
\hline $\begin{array}{l}\text { Studies with no risk of bias according to our risk of } \\
\text { bias assessment }\end{array}$ & 5 & 887 & $0.66(0.43-1.03)$ & $20 \%$ \\
\hline Cardiac surgery & 2 & 349 & $0.53(0.22-1.24)$ & $1 \%$ \\
\hline Mechanical ventilation & 0 & & & \\
\hline Non-cardiac surgery & 6 & 1589 & $0.53(0.35-0.80)$ & $61 \%$ \\
\hline \multicolumn{5}{|l|}{ Standard sedatives } \\
\hline Overall & 15 & 2463 & $0.63(0.46-0.86)$ & $69 \%$ \\
\hline $\begin{array}{l}\text { Studies with no risk of bias according to our risk of } \\
\text { bias assessment }\end{array}$ & 2 & $998^{a^{*}}$ & $0.64(0.41-0.98)$ & $0 \%$ \\
\hline Cardiac surgery & 5 & 509 & $0.40(0.23-0.69)$ & $11 \%$ \\
\hline Mechanical ventilation & 7 & 1536 & $0.82(0.60-1.11)$ & $66 \%$ \\
\hline Non-cardiac surgery ${ }^{b}$ & 2 & 356 & $0.46(0.23-0.90)$ & NA \\
\hline Trim-and-fill method & $20^{c}$ & - & $0.77(0.57-1.05)$ & $64 \%$ \\
\hline \multicolumn{5}{|l|}{ Opioids } \\
\hline Overall & 2 & 441 & $0.61(0.44-0.83)$ & $0 \%$ \\
\hline
\end{tabular}

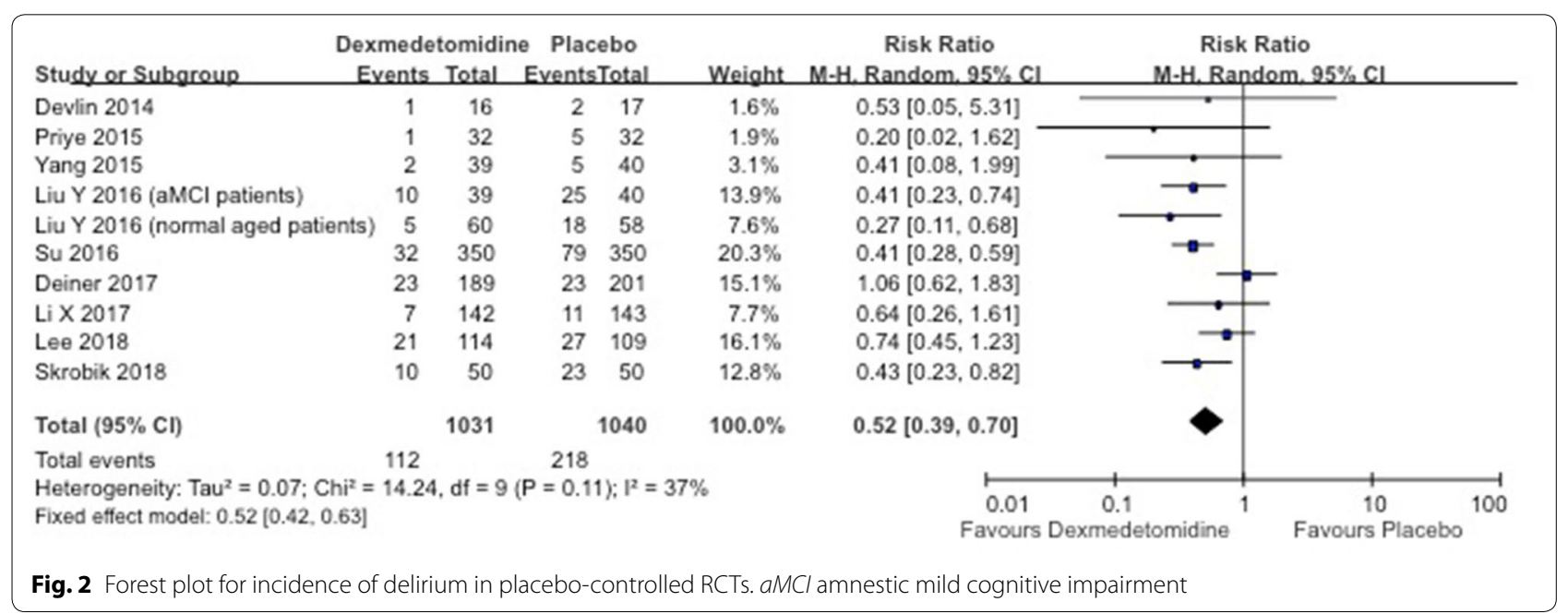

\section{Adverse events}

Explorative analysis of adverse events raising no claim for completeness is presented in Additional file 1: Table S5. When compared to placebo, dexmedetomidine sedation showed a RR of 0.92 for mortality (95\% CI $0.51-1.64$; $\left.I^{2}=0 \%\right)[33,35,45,48,56]$ and a RR of 0.80 for circulatory insufficiency (95\% CI 0.32-2.00; Additional file 1: Table S5) [48]. The risks of bradycardia [33-35, 45, 48, $56]$ and hypotension [33-35, 45, 48, 56] were significantly higher in the dexmedetomidine group, as opposed to tachycardia [35, 48] and hypertension [33, 48].

When compared to standard sedatives, only risks of bradycardia (RR 2.05; 95\% CI 1.31-3.22; $I^{2}=36 \%$ ) [32, 37, $38,40,44,49,50,52]$ and hypotension (RR 1.26; 95\% CI $\left.1.04-1.54 ; I^{2}=9 \%\right)[37,38,40,42,47,50,52]$ were significantly higher in the dexmedetomidine group.

When compared to opioids, dexmedetomidine showed a RR of 0.48 for mortality (95\% CI 0.09-2.60) [41] and 


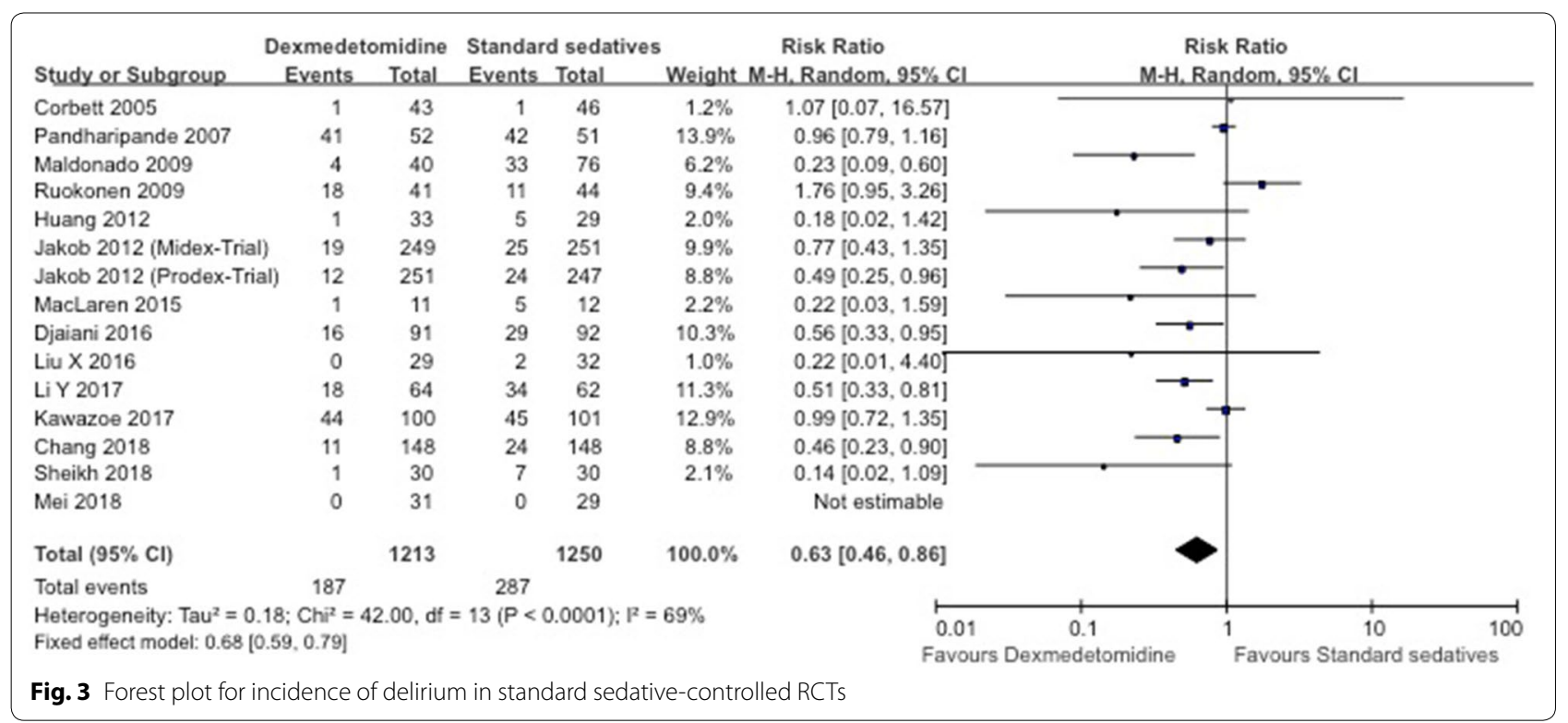

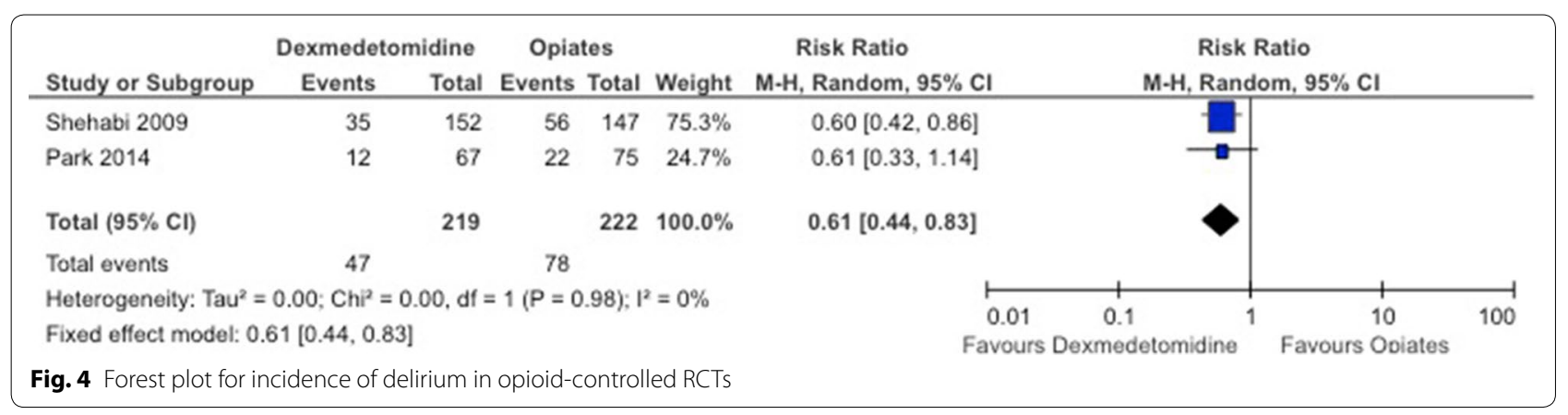

a higher risk of bradycardia with a RR of 2.03 (95\% CI $1.07-3.83 ; I^{2}=22 \%$ ) $[36,41]$.

\section{Clinical outcomes}

Explorative analysis of clinical outcomes raising no claim for completeness is presented in Additional file 1: Table S6. Two outcome measures (i.e., ICU length of stay and time to extubation or duration of mechanical ventilation) were estimated for dexmedetomidine compared to placebo, standard sedatives, and opioids with overall results in favor of dexmedetomidine (Additional file 1: Table S6).

\section{Treatment comparison}

Three RCTs were identified, which reported different clinical outcomes (Additional file 1: Table S7). Control drugs were placebo [57], midazolam [58], and haloperidol [59], each in one RCT. Comparing dexmedetomidine to placebo, Reade et al. [59] found a significantly accelerated resolution of delirium in patients receiving dexmedetomidine (median, $23.3 \mathrm{~h} ; n=39$ vs. $40.0 \mathrm{~h}$; $n=32$; median difference between groups, $16.0 \mathrm{~h}[95 \%$ CI 3.0-28.0 h]; $P=0.01$ ). The risk of bias according to our assessment was low in this study. Using midazolam as control medication, Yapici et al. (2011) who assessed delirium based on CAM-ICU at $36 \mathrm{~h}$ and $60 \mathrm{~h}$ postoperatively had an unclear risk of bias in all five domains. While after $36 \mathrm{~h}$ all patient still had a delirium (i.e., 37 dexmedetomidine treated and 34 midazolam treated patients), they stated significantly lower number of patients with delirium in the intervention group $60 \mathrm{~h}$ postoperatively ( $1 / 37$ patients; $2.7 \%$ vs. $7 / 34$ patients; $21 \%$; $P<0.05)$. Comparing dexmedetomidine to haloperidol, Reade et al. [59] measured proportion of time with satisfactory ICDSC below 4 and desirable ICDSC below 1 as clinical outcome. Patients who received dexmedetomidine "tended to spend a greater proportion of time with satisfactory scores" (median [IQR], 95.5\% [51-100\%] vs. $31.5 \%$ [17-97\%]; $P=0.122$ and $61 \%$ [0-100\%] vs. $0 \%$ 
[0-0\%]; $P=0.134$, respectively) [59]. The RCT was a nonblinded pilot study (including 10 patients in each treatment arm) which had to goal to assess the feasibility of the trial design and the safety of the two treatments.

\section{Discussion}

This meta-analysis suggests dexmedetomidine to be an efficient and reliable sedative agent to reduce the incidence of delirium in critically ill patients when compared to placebo, standard sedatives, and opioids. Our results indicate that the overall incidence of delirium in the dexmedetomidine group was significantly lower compared to placebo, standard sedatives, and opioids. The 2009 study by Maldonado et al. [39] proposed two theories to explain the decreased rates of delirium associated with sedation of dexmedetomidine: The first theory is based on the intrinsic delirium-sparing property determined by multiple characteristics of dexmedetomidine. Since GABA, the primary inhibitory neurotransmitter in the central nervous system seems to play a key role in the pathogenesis of delirium, it is plausible that GABAergic agents such as benzodiazepines and propofol are strongly involved in the development and prolongation of delirium [60,61]. The second theory is that dexmedetomidine-induced sedation provides a more natural sleep-like sedation pattern, which might reduce the risk of developing delirium [19, 62]. Furthermore, dexmedetomidine has very little effect on the cholinergic system, which is strongly linked to cognitive functions and the development of delirium [63]. Finally, when used as an alternative to sedation with GABAergic agents and/or opioids, dexmedetomidine may decrease delirium incidence.

When analyzing cardiac and non-cardiac surgery patients separately, the risk ratios for placebo and standard sedatives seem to be similar to the overall results. As the number of included data sets becomes small in all sensitivity-and subgroup analyses, the results have to be interpreted with caution.

Reduction of overall delirium risk by dexmedetomidine compared to standard sedatives confirms the findings from a meta-analysis conducted and published by Xia et al. [22]. Moreover, a 2014 meta-analysis by Pasin et al. [20] reported that dexmedetomidine significantly decreased delirium incidence when compared to control patients and in a subgroup analysis comparing dexmedetomidine to midazolam. A subgroup of RCTs assessing only cardiac surgery patients showed also results favoring dexmedetomidine. This finding supports Herr et al.s [64] statement, suggesting a potentially positive effect of dexmedetomidine on the incidence of ICU delirium when compared to standard sedatives. The only article [38] with a low risk of bias included two data sets which have similar results when compared to the 12 included data sets.

When compared to opioids, the overall risk of delirium seems to be significantly lower favoring dexmedetomidine. The result has to be interpreted with caution because the opioid-controlled group in this meta-analysis consists of only two RCTs with unclear risk of bias [36, 41].

Of note, it can be interpreted from the subgroup and sensitivity analysis that all data sets point into the same direction, but there are not many studies of high level of evidence estimated by the five dimensions of the risk of bias assessment.

Our additional explorative meta-analyses assessing clinical outcomes confirmed that the use of dexmedetomidine significantly increased the risks of bradycardia and hypotension [32-38, 40-42, 44, 45, 47, 48, 50]. However, we had only limited data to assess the mortality risk or risk of circulatory insufficiency. Large uncertainties exist as indicated by wide $95 \%$ CIs. Here a comprehensive meta-analysis for this endpoint is warranted.

For the treatment of delirium, we identified only three RCTs including all different comparators. Therefore, it is difficult to draw any conclusion about the potential of dexmedetomidine in the treatment of delirium. This systematic review demonstrated the essential need for a standardized measurement for the clinical recovery from delirium. Otherwise, it is impossible to meta-analyze subsequent RCTs on this topic.

Several limitations in our study need to be acknowledged. First, since we focused on incidence of delirium as our primary outcome, we excluded all RCTs not containing delirium data. Of these studies, some might probably have evaluated adverse events and clinical outcome. Therefore, our meta-analysis of adverse events and clinical outcome raises no claim to completeness. Second, we only search in PubMed and CENTRAL for articles published in English. Therefore, it is possible that we missed relevant RCTs because they were published in other languages or published in journals which are not indexed in PubMed or CENTRAL. However, a recently published meta-epidemiological study showed that searching only PubMed and CENTRAL leads in the vast majority to the same conclusion (eventually with less certainty) when compared to more comprehensive Cochrane reviews [65]. Third, only seven out of 28 RCTs could be classified as low risk of bias RCTs. Fourth, there were many significant variations across all RCTs, potentially affecting their comparability. These include different ICU populations, severities of illness, and sedation protocols, leading to heterogeneity in drug doses and potentially influencing outcomes, or different durations of drug administration. Fifth, publication bias could not be assessed in one of the 
three main comparisons due to the limited number of studies [29]. The found effect for the comparison between standard sedatives and dexmedetomidine seemed to be affected by publication bias. Therefore, the results from the trim-and-fill method might be more valid. A further potential limitation, common in all sedation trials, is a masking of differences in sedation if opioids have been administered for clinical reasons [40].

Overall, our systematic review and meta-analysis are the first to focus on the role of dexmedetomidine in the prevention and treatment of the ICU delirium without making any restrictions concerning comparator drugs or types of ICU population. It uses a systematic approach to evaluate the hypothesis that dexmedetomidine is more effective than common therapeutic strategies for the treatment and prevention of delirium.

The results of our meta-analysis suggest that dexmedetomidine may be more efficient in reducing ICU delirium incidence than placebo standard sedatives and opioids. The evidence on treatment of ICU delirium with dexmedetomidine is limited, as only three trials with different comparators each were suitable for our investigation, and needs to be further investigated [66]. Additionally, it is necessary to standardize clinical outcomes in general, and especially in the treatment section to facilitate meta-analyses, thereby ensuring robust evidence.

\section{Additional files}

Additional file 1. Detailed search strategy of electronic database search of PubMed and the Cochrane Central Register of Controlled Trials (CENTRAL).

Additional file 2: Table S4. Table of drug doses used throughout the analyzed studies.

\section{Abbreviations}

CAM: confusion assessment method; CENTRAL: Cochrane Central Register of Controlled Trials; Cl: confidence interval; GABA: gamma-amino-butyric acid; ICDSC: intensive care delirium screening checklist; ICU: intensive care unit; IQR: interquartile range; MV: mechanical ventilation; OR: odds ratio; PAD: pain, agitation, and delirium; RCT: randomized controlled trial; SD: standard deviation.

\section{Authors' contributions}

JF, AH, MS contributed to study concept and design; JF, AH, BS, MS took part in acquisition of data including risk of bias assessment; JF, AH, BS, MS involved in analysis and interpretation of data; JF, AH, MS took part in drafting of the manuscript; all declared authors participated in critical revision of the manuscript for important intellectual content; administrative, technical, or material support is not applicable; MS involved in study supervision. All authors read and approved the final manuscript.

\section{Authors' information}

Julian Flükiger works as a MD candidate at the University Hospital Basel. Alexa Hollinger works as a postdoctoral research fellow at the Saint Louis Lariboisière University Hospitals. Benjamin Speich works as a postdoctoral research at the Centre for Statistics in Medicine, Nuffield Department of Orthopaedics, Rheumatology and Musculoskeletal Sciences, University of Oxford, UK. Vera Meier,
Janna Tontsch, and Tatjana Zehnder are medical students at the University of Basel and are all part of the research team of the Department for Anesthesia, Surgical Intensive Care, Prehospital Emergency Medicine and Pain Therapy of the University Hospital Basel. Martin Siegemund works as deputy head of the surgical intensive care unit at the University Hospital Basel.

\section{Author details}

${ }^{1}$ Department for Anesthesia, Surgical Intensive Care, Prehospital Emergency Medicine and Pain Therapy, University Hospital Basel, Spitalstrasse 21, 4031 Basel, Switzerland. ${ }^{2}$ Department of Anaesthesiology, Burn and Critical Care Medicine, AP-HP, Saint Louis and Lariboisière University Hospitals, 2 rue Ambroise Paré, 75010 Paris, France. ${ }^{3}$ Inserm 942 Paris, Biomarqueurs et maladies cardiaques, Hôpital Lariboisière - Bâtiment Viggo Petersen, 41, boulevard de la Chapelle, 75475 Paris Cedex 10, France. ${ }^{4}$ Department of Clinical Research, Basel Institute for Clinical Epidemiology and Biostatistics, University of Basel and University Hospital Basel, Basel, Switzerland. ${ }^{5}$ Nuffield Department of Orthopaedics, Rheumatology and Musculoskeletal Sciences, Centre for Statistics in Medicine, University of Oxford, Oxford, UK.

\section{Acknowledgements}

The authors thank Allison Dwileski, for providing us with the literature and for proofreading the final manuscript.

\section{Competing interests}

The authors declare that they have no competing interests.

\section{Availability of data and materials}

No review protocol for this systematic review and meta-analysis was registered online. Prof. Siegemund had full access to all data in the study and takes responsibility for the integrity of the data and the accuracy of the data analysis.

\section{Consent for publication \\ Not applicable.}

Ethical approval and consent to participate Not applicable.

Funding

Not applicable.

\section{Publisher's Note}

Springer Nature remains neutral with regard to jurisdictional claims in published maps and institutional affiliations.

Received: 16 June 2018 Accepted: 6 September 2018

Published online: 20 September 2018

\section{References}

1. Barr J, Fraser GL, Puntillo K, Ely EW, Gelinas C, Dasta JF, et al. Clinical practice guidelines for the management of pain, agitation, and delirium in adult patients in the intensive care unit. Crit Care Med. 2013:41(1):263-306.

2. Pandharipande $P$, Cotton BA, Shintani A, Thompson J, Pun BT, Morris JA, et al. Prevalence and risk factors for development of delirium in surgical and trauma intensive care unit patients. J Trauma. 2008;65:34-41.

3. McPherson JA, Wagner CE, Boehm LM, Hall JD, Johnson DC, Miller LR, et al. Delirium in the cardiovascular ICU: exploring modifiable risk factors. Crit Care Med. 2013:41:405-13.

4. Ely EW, Shintani A, Truman B, Speroff T, Gordon S, Harrell F, et al. Delirium as a predictor of mortality in mechanically ventilated patients in the intensive care unit. JAMA J Am Med Assoc. 2004;291:1753-62.

5. Lonergan E, Luxenberg J, Sastre AA, Wyller TB. Benzodiazepines for delirium. Cochrane Database Syst Rev. 2009;1:CD006379.

6. Pandharipande PP, Ely EW, Arora RC, Balas MC, Boustani MA, La Calle GH, et al. The intensive care delirium research agenda: a multinational, interprofessional perspective. Intensive Care Med. 2017;43(9):1329-39. https:// doi.org/10.1007/s00134-017-4860-7. 
7. Hollinger A, Siegemund M, Goettel N, Steiner LA. Postoperative delirium in cardiac surgery: an unavoidable menace? J Cardiothorac Vasc Anesth. 2015;29(6):1677-87

8. Inouye SK, Viscoli CM, Horwitz RI, Hurst LD, Tinetti ME. A predictive model for delirium in hospitalized elderly medical patients based on admission characteristics. Ann Intern Med. 1993;119:474-81.

9. Inouye SK, Charpentier PA. Precipitating factors for delirium in hospitalized elderly persons. JAMA J Am Med Assoc. 1996;275:852-7.

10. Tan JA, Ho KM. Use of dexmedetomidine as a sedative and analgesic agent in critically ill adult patients: a meta-analysis. Intensive Care Med. 2010;36:926-39.

11. Riker RR, Fraser GL. Altering intensive care sedation paradigms to improve patient outcomes. Anesthesiol Clin. 2011;29:663-74.

12. Mantz J, Josserand J, Hamada S. Dexmedetomidine: new insights. Eur J Anaesthesiol. 2011;28:3-6.

13. Girard TD, Pandharipande PP, Ely EW. Delirium in the intensive care unit. Crit Care. 2008;12:S3.

14. Mo Y, Zimmermann AE. Role of dexmedetomidine for the prevention and treatment of delirium in intensive care unit patients. Ann Pharmacother. 2013;47:869-76

15. Barr J, Fraser GL, Puntillo K, Ely EW, Gélinas C, Dasta JF, et al. Clinical practice guidelines for the management of pain, agitation, and delirium in adult patients in the intensive care unit. Crit Care Med. 2013;41:263-306

16. Chen K, Lu Z, Xin Y, Cai Y, Chen Y, Pan S. Alpha-2 agonists for long-term sedation during mechanical ventilation in critically ill patients. Cochrane Database Syst Rev. 2015;1:CD010269.

17. Paris A, Tonner PH. Dexmedetomidine in anaesthesia. Curr Opin Anaesthesiol. 2005;18:412-8.

18. Huupponen E, Maksimow A, Lapinlampi P, Sarkela M, Saastamoinen A Snapir A, et al. Electroencephalogram spindle activity during dexmedetomidine sedation and physiological sleep. Acta Anaesthesiol Scand. 2008;52(2):289-94.

19. Venn RM, Bradshaw CJ, Spencer R, Brealey D, Caudwell E, Naughton C, et al. Preliminary UK experience of dexmedetomidine, a novel agent for postoperative sedation in the intensive care unit. Anaesthesia. 1999:54:1136-42

20. Pasin L, Landoni G, Nardelli P, Belletti A, Di Prima AL, Taddeo D, et al. Dexmedetomidine reduces the risk of delirium, agitation and confusion in critically III patients: a meta-analysis of randomized controlled trials. J Cardiothorac Vasc Anesth. 2014;28(6):1459-66.

21. Szumita PM, Baroletti SA, Anger KE, Wechsler ME. Sedation and analgesia in the intensive care unit: evaluating the role of dexmedetomidine. Am J Health Syst Pharm AJHP. 2007;64:37-44.

22. Xia Z-Q, Chen S-Q, Yao X, Xie C-B, Wen S-H, Liu K-X. Clinical benefits of dexmedetomidine versus propofol in adult intensive care unit patients: a meta-analysis of randomized clinical trials. J Surg Res. 2013;185:833-43.

23. Duan X, Coburn M, Rossaint R, Sanders RD, Waesberghe JV, Kowark A. Efficacy of perioperative dexmedetomidine on postoperative delirium: systematic review and meta-analysis with trial sequential analysis of randomised controlled trials. Br J Anaesth. 2018;121(2):384-97.

24. Liberati A, Altman DG, Tetzlaff J, Mulrow C, Gøtzsche PC, loannidis JPA, et al. The PRISMA statement for reporting systematic reviews and metaanalyses of studies that evaluate health care interventions: explanation and elaboration. PLoS Med. 2009;6:e1000100.

25. Higgins JPT, Altman DG, Gotzsche PC, Juni P, Moher D, Oxman AD, et al. The Cochrane Collaboration's tool for assessing risk of bias in randomised trials. BMJ. 2011;343:d5928.

26. Duval S, Tweedie R. Trim and fill: a simple funnel-plot-based method of testing and adjusting for publication bias in meta-analysis. Biometrics. 2000;56(2):455-63.

27. Duval STRA. Nonparametric "trim and fill" method of accounting for publication bias in meta-analysis. J Am Stat Assoc. 2000;95(449):89-98.

28. Higgins JPT, Thompson SG. Quantifying heterogeneity in a meta-analysis. Stat Med. 2002;21:1539-58.

29. Higgins J, Green S. Cochrane handbook for systematic reviews of interventions. London: The Cochrane Collaboration; 2011.

30. Wan X, Wang W, Liu J, Tong T. Estimating the sample mean and standard deviation from the sample size, median, range and/or interquartile range. BMC Med Res Methodol. 2014;14:135.
31. Luo D, Wan X, Liu J, Tong T. Optimally estimating the sample mean from the sample size, median, mid-range and/or mid-quartile range. Stat Methods Med Res. 2016:27:1785.

32. Pandharipande PP, Pun BT, Herr DL, Maze M, Girard TD, Miller RR, et al. Effect of sedation with dexmedetomidine vs lorazepam on acute brain dysfunction in mechanically ventilated patients: the MENDS randomized controlled trial. JAMA. 2007;298:2644-53.

33. Deiner S, Luo X, Lin H-M, Sessler DI, Saager L, Sieber FE, et al. Intraoperative infusion of dexmedetomidine for prevention of postoperative delirium and cognitive dysfunction in elderly patients undergoing major elective noncardiac surgery. JAMA Surg. 2017;152:e171505.

34. Yang X, Li Z, Gao C, Liu R. Effect of dexmedetomidine on preventing agitation and delirium after microvascular free flap surgery: a randomized, double-blind, control study. J Oral Maxillofac Surg. 2015;73:1065-72.

35. Devlin JW, Al-Qadheeb NS, Chi A, Roberts RJ, Qawi I, Garpestad E, et al. Efficacy and safety of early dexmedetomidine during noninvasive ventilation for patients with acute respiratory failure: a randomized, doubleblind, placebo-controlled pilot study. Chest. 2014;145:1204-12.

36. Park JB, Bang SH, Chee HK, Kim JS, Lee SA, Shin JK. Efficacy and safety of dexmedetomidine for postoperative delirium in adult cardiac surgery on cardiopulmonary bypass. Korean J Thorac Cardiovasc Surg. 2014;47(3):249-54

37. Huang Z, Chen Y-S, Yang Z-L, Liu J-Y. Dexmedetomidine versus midazolam for the sedation of patients with non-invasive ventilation failure. Intern Med. 2012:51:2299-305.

38. Jakob SM, Ruokonen E, Grounds RM, Sarapohja T, Garratt C, Pocock SJ, et al. Dexmedetomidine vs midazolam or propofol for sedation during prolonged mechanical ventilation. JAMA. 2012;307:1151-60.

39. Maldonado JR, Wysong A, van der Starre PJ, Block T, Miller C, Reitz BA Dexmedetomidine and the reduction of postoperative delirium after cardiac surgery. Psychosomatics. 2009:50(3):206-17.

40. Ruokonen E, Parviainen I, Jakob SM, Nunes S, Kaukonen M, Shepherd ST, et al. Dexmedetomidine versus propofol/midazolam for long-term sedation during mechanical ventilation. Intensive Care Med. 2009;35:282-90.

41. Shehabi Y, Grant P, Wolfenden H, Hammond N, Bass F, Campbell M, et al. Prevalence of delirium with dexmedetomidine compared with morphine based therapy after cardiac surgery: a randomized controlled trial (DEXmedetomidine COmpared to Morphine-DEXCOM Study). Anesthesiology. 2009;111(5):1075-84.

42. Corbett SM, Rebuck JA, Greene CM, Callas PW, Neale BW, Healey MA, et al. Dexmedetomidine does not improve patient satisfaction when compared with propofol during mechanical ventilation. Crit Care Med. 2005;33:940-5

43. Li Y, Yu Z-X, Ji M-S, Yan J, Cai Y, Liu J, et al. A pilot study of the use of dexmedetomidine for the control of delirium by reducing the serum concentrations of brain-derived neurotrophic factor, neuron-specific enolase, and S100B in polytrauma patients. J Intensive Care Med. 2017;1:885066617710643

44. Kawazoe Y, Miyamoto K, Morimoto T, Yamamoto T, Fuke A, Hashimoto $A$, et al. Effect of dexmedetomidine on mortality and ventilator-free days in patients requiring mechanical ventilation with sepsis. JAMA 2017;317:1321-8.

45. Li X, Yang J, Nie X-L, Zhang Y, Li X-Y, Li L-H, et al. Impact of dexmedetomidine on the incidence of delirium in elderly patients after cardiac surgery: a randomized controlled trial. PLOS ONE. 2017;12:e0170757.

46. Djaiani G, Silverton N, Fedorko L, Carroll J, Styra R, Rao V, et al. Dexmedetomidine versus propofol sedation reduces delirium after cardiac surgery. Anesthesiology. 2016;124:362-8.

47. Liu X, Zhang K, Wang W, Xie G, Cheng B, Wang Y, et al. Dexmedetomidine versus propofol sedation improves sublingual microcirculation after cardiac surgery: a randomized controlled trial. J Cardiothorac Vasc Anesth. 2016:30:1509-15.

48. Su X, Meng Z-T, Wu X-H, Cui F, Li H-L, Wang D-X, et al. Dexmedetomidine for prevention of delirium in elderly patients after non-cardiac surgery: a randomised, double-blind, placebo-controlled trial. Lancet. 2016:388:1893-902

49. Liu Y, Ma L, Gao M, Guo W, Ma Y. Dexmedetomidine reduces postoperative delirium after joint replacement in elderly patients with mild cognitive impairment. Aging Clin Exp Res. 2016;28:729-36.

50. MacLaren R, Preslaski CR, Mueller SW, Kiser TH, Fish DN, Lavelle JC, et al. A randomized, double-blind pilot study of dexmedetomidine versus 
midazolam for intensive care unit sedation. J Intensive Care Med. 2015;30:167-75

51. Priye S, Jagannath S, Singh D, Shivaprakash S, Reddy D. Dexmedetomidine as an adjunct in postoperative analgesia following cardiac surgery: a randomized, double-blind study. Saudi J Anaesth. 2015;9:353-8.

52. Chang YF, Chao A, Shih PY, Hsu YC, Lee CT, Tien YW, et al. Comparison of dexmedetomidine versus propofol on hemodynamics in surgical critically ill patients. J Surg Res. 2018;228:194-200.

53. Sheikh TA, Dar BA, Akhter N, Ahmad N. A comparative study evaluating effects of intravenous sedation by dexmedetomidine and propofol on patient hemodynamics and postoperative outcomes in cardiac surgery. Anesth Essays Res. 2018;12(2):555-60.

54. Mei B, Meng G, Xu G, Cheng X, Chen S, Zhang Y, et al. Intraoperative sedation with dexmedetomidine is superior to propofol for elderly patients undergoing hip arthroplasty: a prospective randomized controlled study. Clin J Pain. 2018;34(9):811-7.

55. Lee $\mathrm{C}$, Lee $\mathrm{CH}$, Lee $\mathrm{G}$, Lee $\mathrm{M}$, Hwang J. The effect of the timing and dose of dexmedetomidine on postoperative delirium in elderly patients after laparoscopic major non-cardiac surgery: a double blind randomized controlled study. J Clin Anesth. 2018:47:27-32.

56. Skrobik Y, Duprey MS, Hill NS, Devlin JW. Low-dose nocturnal dexmedetomidine prevents ICU delirium. A randomized, placebo-controlled trial. Am J Respir Crit Care Med. 2018;197(9):1147-56.

57. Reade MC, Eastwood GM, Bellomo R, Bailey M, Bersten A, Cheung B, et al. Effect of dexmedetomidine added to standard care on ventilator-free time in patients with agitated delirium. JAMA. 2016;315:1460-8.

58. Yapici N, Coruh T, Kehlibar T, Yapici F, Tarhan A, Can Y, et al. Dexmedetomidine in cardiac surgery patients who fail extubation and present with a delirium state. Heart Surg Forum. 2011;14:E93-8.
59. Reade MC, O'Sullivan K, Bates S, Goldsmith D, Ainslie WR, Bellomo R. Dexmedetomidine vs. haloperidol in delirious, agitated, intubated patients: a randomised open-label trial. Crit Care. 2009;13(3):R75.

60. Sanders RD, Maze M. Contribution of sedative-hypnotic agents to delirium via modulation of the sleep pathway. Can J Anaesth. 2011;58(2):14956. https://doi.org/10.1007/s12630-010-9421-2.

61. Pandharipande P, Shintani A, Peterson J, Pun BT, Wilkinson GR, Dittus RS et al. Lorazepam is an independent risk factor for transitioning to delirium in intensive care unit patients. Anesthesiology. 2006;104:21-6.

62. Coull JT, Jones MEP, Egan TD, Frith CD, Maze M. Attentional effects of noradrenaline vary with arousal level: selective activation of thalamic pulvinar in humans. Neurolmage. 2004;22:315-22.

63. Zaal IJ, Slooter AJC. Delirium in critically ill patients: epidemiology, pathophysiology, diagnosis and management. Drugs. 2012;72:1457-71.

64. Herr DL, Sum-Ping STJ, England M. ICU sedation after coronary artery bypass graft surgery: dexmedetomidine-based versus propofol-based sedation regimens. J Cardiothorac Vasc Anesth. 2003;17:576-84.

65. Nussbaumer-Streit B, Klerings I, Wagner G, Heise TL, Dobrescu Al, ArmijoOlivo $S$, et al. Abbreviated literature searches were viable alternatives to comprehensive searches: a meta-epidemiological study. J Clin Epidemiol. 2018;102:1.

66. Hollinger A, Ledergerber K, Von Felten S, Sutter R, Rüegg S, Gantner L, et al. Comparison of propofol and dexmedetomidine infused overnight to treat hyperactive and mixed ICU delirium: a protocol for the Basel ProDex clinical trial. BMJ Open. 2017;7:e015783.

\section{Submit your manuscript to a SpringerOpen ${ }^{\odot}$ journal and benefit from:}

- Convenient online submission

- Rigorous peer review

- Open access: articles freely available online

- High visibility within the field

- Retaining the copyright to your article

Submit your next manuscript at $\boldsymbol{\nabla}$ springeropen.com 